\title{
Fünfzehn Minuten für eine noch bessere Weiterbildung: der Fragebogen 2004
}

\author{
Max Giger, Präsident der Kommission für Weiter- und Fortbildung FMH, Winterthur
}

Die Umfrage 2003 erfolgte mit einem neuen Fragebogen. Die Resultate führten an vielen Weiterbildungsstätten zu durchaus erwünschten Diskussionen über die Effizienz der Weiterbildung. Sinn und Gestaltung des Fremdjahres müssen hinterfragt werden. Der überarbeitete Fragebogen 2004 geht nebst den allgemeinen Fragen den Themata Fehlerkultur und evidenzbasierte Medizin in der Weiterbildung nach. sicherung der Weiterbildung. Die Beantwortung des Fragebogens dauert gerade eine Viertelstunde.

Die diesjährige Umfrage wird den zwei Jahresthemata «Vermittlung von Fehlerkultur» und «Vermittlung von Anwendung der evidenzbasierten Medizin» nachgehen. Über erste Resultate hoffen wir im Spätherbst berichten zu können.

Selbstverständlich sollen sich auch dieses Beurteilung der Weiterbildung aus Sicht der Assistenzärztinnen und Assistenzärzte an die Weiterbildungsstätten. Diese schon zum achten Mal erfolgende Umfrage soll wie die früheren ermöglichen, ein Bild der Urteile aus Sicht der Weiterzubildenden erhalten. Eine möglichst zahlreiche Teilnahme der Befragten, um derentwillen die Weiterbildung erfolgt, ist eine wünschenswerte Pflicht für alle, die sich in Weiterbildung zu einem Facharzttitel befinden. Die Umfrage ist eines der drei Mittel zur Qualitäts-
Jahr sowohl Weiterzubildende wie auch Weiterbildner kritisch mit dem Fragebogen auseinandersetzen. Kritische Bemerkungen nehme ich gerne persönlich entgegen oder können auf der Website der AWF (www.fmh.ch/awf) öffentlich zugänglich gemacht werden. Ich bin überzeugt, dass wir mit konstruktiven Kritiken die Qualität des Fragebogens von Jahr zu Jahr verbessern können und damit valide Resultate über den Zustand der Weiterbildungsstätten erhalten werden. 